\title{
Doppler Cooling and Trapping on Forbidden Transitions
}

\author{
T. Binnewies, G. Wilpers, U. Sterr, F. Riehle, and J. Helmcke \\ Physikalisch-Technische Bundesanstalt, 38116 Braunschweig, Germany \\ T. E. Mehlstäubler, E. M. Rasel, and W. Ertmer \\ Institut für Quantenoptik, Universität Hannover, 30167 Hannover, Germany
}

(Received 18 May 2001; published 30 August 2001)

\begin{abstract}
Ultracold atoms at temperatures close to the recoil limit have been achieved by extending Doppler cooling to forbidden transitions. A cloud of ${ }^{40} \mathrm{Ca}$ atoms has been cooled and trapped to a temperature as low as $6 \mu \mathrm{K}$ by operating a magnetooptical trap on the spin-forbidden intercombination transition. Quenching the long-lived excited state with an additional laser enhanced the scattering rate by a factor of 15 , while a high selectivity in velocity was preserved. With this method, more than $10 \%$ of precooled atoms from a standard magnetooptical trap have been transferred to the ultracold trap. Monte Carlo simulations of the cooling process are in good agreement with the experiments.
\end{abstract}

DOI: $10.1103 /$ PhysRevLett.87.123002

PACS numbers: $32.80 . P \mathrm{j}, 42.50 . \mathrm{Vk}$

The preparation of ultracold atoms with temperatures far below the millikelvin regime is essential for progress in many fields such as metrology, atom optics, and the study of collective effects like Bose-Einstein condensation (BEC). Up to now the available techniques have been restricted to a limited number of species, mainly those that can be cooled below the Doppler limit [1], e.g., by efficient polarization gradient cooling [2], by velocity selective coherent population trapping (VSCPT) [3] or by Raman cooling [4]. In general, these techniques are applicable only to atoms with magnetic or hyperfine substructure in the ground state. Atoms with a single ground state, such as the alkaline earths, often exhibit forbidden transitions with unique properties, e.g., for optical frequency standards [5-7], atom interferometry [8], the study of cold collisions [9,10], and possibly for achieving BEC by all optical means [11,12]. Doppler cooling on such forbidden transitions can lead to temperatures much lower than the recoil limit [13]. In strontium Doppler cooling has recently led to phase space densities close to quantum degeneracy [14]. Up to now, this method failed to work for neutral atoms with ultranarrow (forbidden) lines since in this case the light forces might be comparable to or even smaller than the gravitational force which makes magnetooptical trapping of atoms difficult or impossible.

In this Letter we present a novel method for effective Doppler cooling and trapping of precooled atoms on extremely narrow transitions in three dimensions. The low scattering rate associated with the narrow line width is artificially enhanced by a repumping or "quenching" laser which deexcites the atoms via an intermediate level. In this case the atoms can be considered as effective two-level systems with tunable linewidths that can be tailored during the cooling process for optimum efficiency of the procedure. Similar schemes were performed to more rapidly cool trapped ions [15] and for the efficient deflection of a beam of metastable helium [16]. Whereas in the case of metastable helium cycling on the second transition was simply used to increase the radiation pressure, our scheme preserves the velocity selectivity of the narrow transition. Our calculations for magnesium and calcium showed that this "quench cooling" can efficiently trap atoms released from a broad-line magnetooptical trap (MOT) and cool them to temperatures close to the recoil limit. We utilize this scheme for the first time to capture and cool free atoms in three dimensions.

The temperature that can be achieved in Doppler cooling on broad lines is determined by a balance between damping forces and the heating due to spontaneous recoil momenta. Here, broad lines are characterized by the linewidth of the transition $\Gamma$ being large compared to the change in the Doppler shift due to one photon recoil, i.e., $\frac{\hbar k^{2}}{m} \ll \Gamma$ where $m$ denotes the atomic mass and $k$ the wave vector of the exciting light. On a two-level system this finally leads to a Doppler temperature $T_{D}=\frac{\hbar \Gamma}{2 k_{B}}$ with the Boltzmann's constant $k_{B}$.

For monochromatic excitation on narrow lines, the Doppler cooling limit was calculated to be approximately half the recoil temperature given by $k_{B} T_{\mathrm{rec}}=\frac{\hbar^{2} k^{2}}{m}$ [17]. A multichromatic excitation was proposed [13] which would allow us to reach a temperature even below that temperature. However, narrow-line cooling is hampered by the small force $F_{\max }=\frac{\hbar k \Gamma}{2}$. For cooling of calcium on the intercombination line ${ }^{1} S_{0^{-}}{ }^{3} P_{1}$ (see Fig. $1, \Gamma_{1}=2000 \mathrm{~s}^{-1}$ ) $F_{\max }$ is only 1.5 times the gravitational force. To increase the force, we have reduced the lifetime of the upper level through excitation to the $4 s 4 d^{1} D_{2}$ state $(\lambda=453 \mathrm{~nm})$, from where the atoms decay rapidly via the $4 s 4 p{ }^{1} P_{1}$ state to the ground state. In a different picture, the ${ }^{3} P_{1}$ level acquires an admixture of the ${ }^{1} D_{2}$ level (natural decay rate $\Gamma_{2}=1.4 \times 10^{7} \mathrm{~s}^{-1}$ ) through the coupling by the quench laser. Hence, for low saturation the width of the triplet state increases proportional to the quench laser intensity. Another possibility for quenching was demonstrated by 


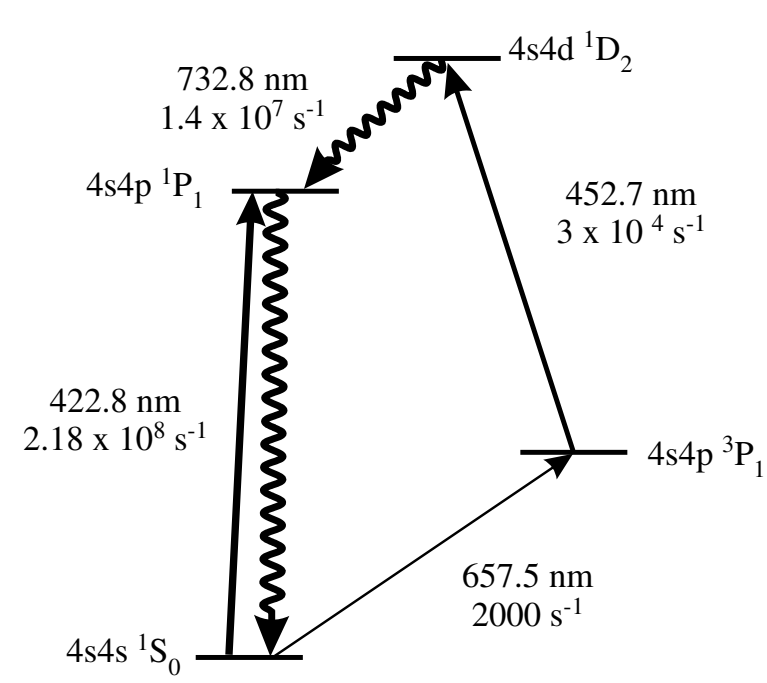

FIG. 1. Excerpt from the Ca level scheme with wavelengths and $A$ coefficients of the relevant transitions. The ${ }^{1} S_{0^{-}}{ }^{1} P_{1}$ transition is used for precooling atoms in a standard broad-line MOT. The intercombination transition ${ }^{1} S_{0}{ }^{3} P_{1}$, quenched by the transition ${ }^{3} P_{1^{-}}{ }^{1} D_{2}$ forms the second stage MOT.

the NIST group where coupling to the $4 s 5 s{ }^{1} S_{0}$ state by a $553 \mathrm{~nm}$ laser was used to achieve cooling of $\mathrm{Ca}$ in one dimension [18].

In a preliminary experiment in 1D with sequential excitation to the ${ }^{3} P_{1}$ state and subsequent quenching we have found a quenching rate of $r_{12} \approx 1.4 \times 10^{4} \mathrm{~s}^{-1}$. This is more than 1 order less than is calculated from the atomic data [19] of the quenching transition $\left(A=3 \times 10^{4} \mathrm{~s}^{-1}\right)$. We assume the difference to be in part due to the uncertainty in the theoretical $A$ coefficient. From our preliminary measurement we estimate a quenching rate $r_{12} \approx$ $3 \times 10^{4} \mathrm{~s}^{-1}$ for our 3D MOT configuration, corresponding to an average broadening factor of 15 limited by the laser power available. In the MOT configuration, excitation to the ${ }^{3} P_{1}$ level and quenching is performed simultaneously. Solving the optical Bloch equations we arrive at an effective two level system with an effective linewidth of the metastable ${ }^{3} P_{1}$ level of $\Gamma_{\text {eff }}=\Gamma_{1}+r_{12}$. Since the increased linewidth of $\Gamma_{\text {eff }} \approx 2 \pi \times 5 \mathrm{kHz}$ is still small compared to the recoil shift, atoms can be lost when kicked out of resonance by the photon recoils of one quenching cycle. To overcome this we broaden the excitation spectrum by a harmonic frequency modulation (peak-topeak amplitude $\delta f_{p p}=1.4 \mathrm{MHz}$, modulation frequency $f_{\text {mod }}=15 \mathrm{kHz}$ ) of the $657-\mathrm{nm}$ laser (linewidth approximately $500 \mathrm{~Hz}$ ) using an acoustooptic modulator. The high-frequency end of this frequency comb is detuned below resonance by $\Delta \approx 2 \pi \times 280 \mathrm{kHz}$.

With the laser power available on the intercombination transition we saturate the transition even with the quenching laser on. With a population in the ${ }^{3} P_{1}$ state close to 0.4 the total scattering rate amounts to $S \approx 1.2 \times 10^{4} \mathrm{~s}^{-1}$.

In the absence of a magnetic field, two cooling regimes can be distinguished: In the Doppler regime which is effective at larger velocities, the Doppler shift brings the atom into resonance with a counterpropagating laser beam. This leads to a constant damping force towards zero velocity and a typical cooling time of $t_{D} \approx m v_{0} / \hbar k_{1} S=$ $5.6 \mathrm{~ms}$ using an initial velocity $v_{0}=1 \mathrm{~m} / \mathrm{s}$ and $k_{1}$ being the wave vector of the $657 \mathrm{~nm}$ laser. Because this time has to be small enough to prevent atoms from escaping the laser beams before being stopped, we have increased the force by using the same circular polarization for the cooling $657 \mathrm{~nm}$ beams and the quenching $453 \mathrm{~nm}$ laser. Absorption of the quenching photon from the same direction as the initial photon is favored by 6 to 1 as a result of the ratio of the Clebsch-Gordan coefficients involved.

The second regime occurs at lower temperatures when slow atoms are not excited by any laser and accumulate near zero velocity. Compared to VSCPT there is still a small off-resonant excitation probability. Therefore this regime can be referred to as "velocity-selective gray-state trapping." The limit of this cooling method depends on the detuning $\Delta$ and can be as low as a fraction of the recoil velocity. The relevant time scale is much longer than in the Doppler regime mentioned above as the cooling relies on random jumps to the gray state.

The situation changes with the magnetic quadrupole field present in the MOT configuration. Here, atoms that are in the gray state at the MOT center will eventually move to a point where they come into resonance with one laser by the Zeeman shift. There they will be pumped out of the gray state and accelerated towards the trap center until the atoms are just Doppler shifted off resonance. Therefore the average atomic velocity will be of the order of a few recoils [20] independent of the detuning $\Delta$. The detuning, however, determines the size of the cold atomic cloud.

The recoil that determines the achievable velocity width is due to the fluorescence photons at 733 and $423 \mathrm{~nm}$ from the decay of the ${ }^{1} D_{2}$ level (Fig. 1) and the quench photon at $453 \mathrm{~nm}$ that are involved in the final cycle before the atom gets off resonance. The effective recoil velocity was chosen as the quadratic sum of the three photon momenta that amounts to $v_{\text {rec }} \approx 3.5 \mathrm{~cm} / \mathrm{s}$. Here we assumed also a random direction of the absorbed quench photon, as we could not observe the expected polarization effect experimentally. This is probably due to the fact that the 657 and $453 \mathrm{~nm}$ beams could not be overlapped in all three directions.

We realized this novel cooling scheme in an experiment, where we started with a broad-line MOT on the cooling transition ${ }^{1} S_{0^{-}}{ }^{1} P_{1}$ made of three retroreflected laser beams with $\lambda=423 \mathrm{~nm}$. With a total power of $30 \mathrm{~mW}$ approximately $10^{7}$ atoms were cooled and trapped directly from a thermal beam. The narrow-line MOT at $657 \mathrm{~nm}$ was realized by three circular polarized beams (power approximately $7 \mathrm{~mW}$ per beam with a diameter of $5 \mathrm{~mm}$ ), that were parallel to the $423 \mathrm{~nm}$ MOT beams. Dichroitic retroreflectors were used to combine the beams. The quench laser beams at $453 \mathrm{~nm}$ were coupled into 
the vacuum chamber under an angle of $22.5^{\circ}$ to the other cooling beams in the horizontal plane and nearly parallel in the vertical direction. To make efficient use of the available power $(P \approx 20 \mathrm{~mW})$ a single beam of diameter $\approx 3 \mathrm{~mm}$ was fed through the setup three times and finally retroreflected, similar to a MOT configuration. In our vacuum chamber it was not possible to completely match the sizes and directions of the quench and MOT laser beams. We expect this to limit the possible transfer efficiency in our setup.

After precooling the atoms in the broad-line MOT to $v_{\text {rms }} \approx 0.7 \mathrm{~m} / \mathrm{s}$, the $423 \mathrm{~nm}$ laser was switched off and the magnetic field gradient was lowered within $100 \mu \mathrm{s}$ from $60 \times 10^{-4}$ to $0.3 \times 10^{-4} \mathrm{~T} / \mathrm{cm}$ and the $657 \mathrm{~nm}$ cooling laser and the quench laser were switched on. The small gradient accounts for the much smaller acceleration that can be reached by cooling on the narrow line, even with quenching. After the cooling time the $657 \mathrm{~nm}$ laser was switched off while the quench laser remained on to repump most of the atoms to the ground state. To reduce the background from fluorescence of decaying atoms that have not been repumped, we waited for 0.5 to $1 \mathrm{~ms}$ before measuring the velocity distribution.

The velocity distribution of the atomic ensemble was obtained by measuring the Doppler broadening of the intercombination transition. To avoid Zeeman broadening of the transition the magnetic field was changed from a quadrupole to a homogeneous field. Figure 2 shows a typical velocity distribution detected from the fluorescent decay of the ${ }^{3} P_{1}$ state before and after the cooling on the quenched intercombination line. Comparing the areas of the two curves in Fig. 2 we find a transfer efficiency of $\eta_{N}=12 \%$. Because of the integration over all transverse velocities the increase in count rate by a factor of 2 shown in Fig. 2, does not reflect the increase in the number of

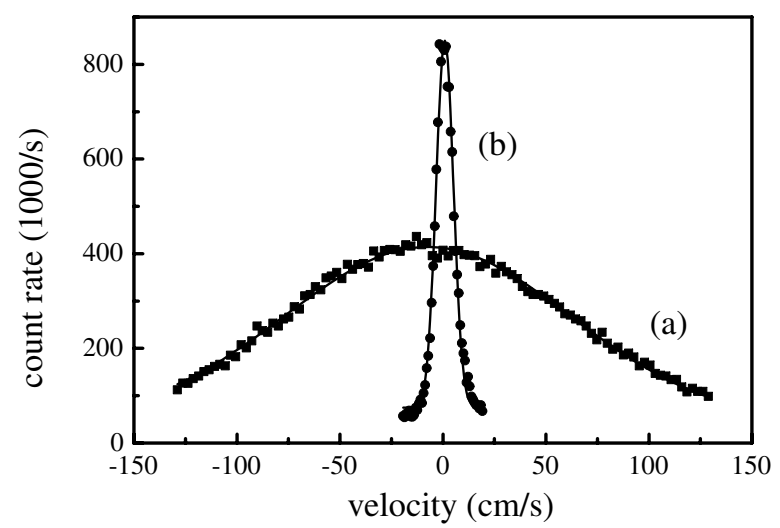

FIG. 2. Velocity distribution measured by the excitation spectrum of the Doppler broadened intercombination transition. The distribution after cooling in a usual broad-line MOT (a) shows an $\mathrm{rms}$ velocity of $71 \mathrm{~cm} / \mathrm{s}(T=2.4 \mathrm{mK})$ which is reduced to $4.3 \mathrm{~cm} / \mathrm{s}(T=9 \mu \mathrm{K})$ after $25 \mathrm{~ms}$ of second stage cooling (b). Comparing the areas of the two distributions gives a transfer efficiency of $12 \%$. atoms $\eta_{v}$ with $v \approx 0$. This increase is calculated from $\eta_{v} \eta_{N}\left(v_{\mathrm{rms}, 423} / v_{\mathrm{rms}, 657}\right)^{3}$ as $\eta_{v} \approx 500$.

The temperature as a function of the cooling time (Fig. 3) shows an exponential decay with a time constant of $1.4 \mathrm{~ms}$ until the rms velocity is reduced to a few times the effective recoil velocity $v_{\text {rec }}$. For longer cooling times a further slow reduction of the velocity width is observed. A temperature of $(5.6 \pm 0.4) \mu \mathrm{K}$ after $100 \mathrm{~ms}$ of cooling has been observed, which corresponds to an rms velocity of $3.4 \mathrm{~cm} / \mathrm{s}$ that is close to the calculated effective $v_{\text {rec }}$. The time dependence of the total number of atoms as deduced from the area of the measured Doppler curves (Fig. 3) shows first a fast loss due to atoms that could not be captured by the narrow-line MOT and then an almost linear decrease in atom number with cooling time. From absorption images of the ultracold atomic cloud after a cooling time of 15 to $20 \mathrm{~ms}$ we obtain rms radii of 0.8 and $0.4 \mathrm{~mm}$ in the horizontal and vertical directions, respectively.

In our present setup significant variations of the final temperature and the transfer efficiency occur over the day as can be seen from the different scales of Figs. 3 and 4. We attribute this to slight changes in the alignment and the detuning of the quench laser.

We have modeled the cooling and trapping by a semiclassical 3D Monte-Carlo simulation. The temporal dependence of the temperature is in good agreement with the experimental results also showing a fast reduction and a further slow decrease of the rms velocity approaching the recoil velocity for long time $s$. The calculated transfer efficiency typically amounts to $60 \%$, far above the experimentally observed $12 \%$, probably due to limitations of our setup as mentioned above. The slow loss of atoms also shows up in the Monte-Carlo simulations, where it was identified as being caused by atoms moving out of the

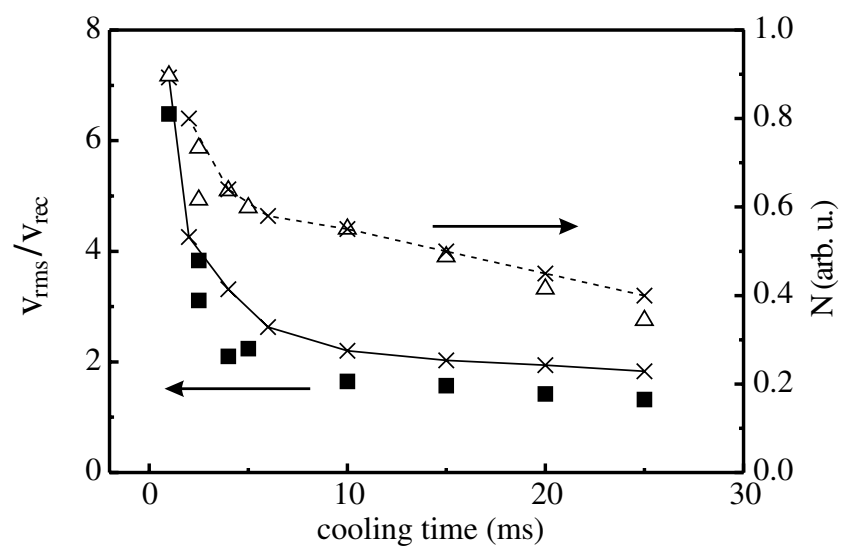

FIG. 3. Atom numbers (triangles) and root-mean-square velocities $v_{\text {rms }}$ (squares) in units of the effective recoil velocity $v_{\text {rec }}=3.5 \mathrm{~cm} / \mathrm{s}$ after second stage cooling versus the cooling time. The crosses connected by lines show the results of Monte Carlo simulations using the experimental parameters. The simulated atom number is scaled to fit the measurement. 


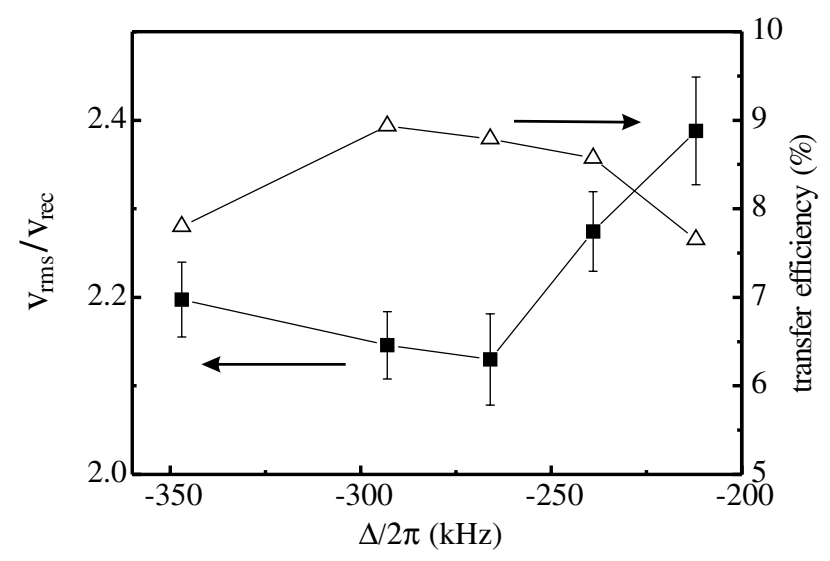

FIG. 4. Root-mean-square velocity and transfer efficiency after $25 \mathrm{~ms}$ of second stage cooling versus the detuning of the $657 \mathrm{~nm}$ MOT laser. The bars indicate the statistical uncertainty of $v_{\text {rms }}$ from the fits to the measured velocity distributions.

intersection region of the laser beams. Similar temperatures near the recoil limit have been obtained with simulations for the narrow-line cooling of magnesium on the intercombination transition $3 s^{2}{ }^{1} S_{0}-3 s 3 p^{3} P_{1}$ and quenching using the $3 s 3 p^{3} P_{1}-3 s 4 s^{1} S_{0}$ transition.

The detuning dependence of the final temperature is shown in Fig. 4. The velocity reaches a minimum around $270 \mathrm{kHz}$ and is nearly constant for larger detunings, as discussed above. The increase at lower detunings is probably due to excitation in the wings of the excitation profile. The transfer efficiency shows a maximum at approximately the same detuning, as for smaller detunings the capturing probability is reduced. For larger detunings the loss increases because the trap becomes larger than the intersection region of the laser beams.

This quench cooling scheme has the distinct advantage that the scattering rate can be tailored for different purposes by adjusting the power of the quench laser, e.g., using a high scattering rate for efficient capturing and then lowering the rate to reach the lowest temperatures. For use in an atomic frequency standard ultracold atoms at moderate density are desired. This can be reached by completely switching off the magnetic field and finally cooling the atoms in an optical molasses to reach even subrecoil temperatures. On the other hand, to enable efficient filling of an optical dipole trap one would increase the magnetic field gradient after a few milliseconds to reach a high phase-space density.

The presented results open the way to a wide variety of experiments including the formation of quantum degenerate ensembles by all-optical means. This might prove par- ticularly useful in the case of $\mathrm{Ca}$ where calculations [12] indicate a positive scattering length.

In conclusion, we have presented a cooling method applicable to atoms with narrow or forbidden transitions. We have demonstrated this method by cooling a ${ }^{40} \mathrm{Ca}$ atomic ensemble in three dimensions to a residual rms velocity close to one effective recoil. Our scheme is applicable to arbitrarily narrow atomic transitions that can be artificially broadened and, hence, does not suffer from the associated small forces.

We thank J. Keupp and S. Baluchev for technical assistance and M. Lewenstein for helpful discussions. The work was supported by the Deutsche Forschungsgemeinschaft under SFB 407 and by the European Commission through the Human Potential Programme (Cold Atoms and Ultra-precise Atomic Clocks; CAUAC).

[1] S. Chu et al., Phys. Rev. Lett. 55, 48 (1985).

[2] P. D. Lett et al., Phys. Rev. Lett. 61, 169 (1988).

[3] A. Aspect et al., Phys. Rev. Lett. 61, 826 (1988).

[4] M. Kasevich and S. Chu, Phys. Rev. Lett. 69, 1741 (1992).

[5] F. Ruschewitz et al., Phys. Rev. Lett. 80, 3173 (1998).

[6] C. W. Oates, F. Bondu, R. W. Fox, and L. Hollberg, Eur. Phys. J. D 7, 449 (1999).

[7] F. Riehle et al., IEEE Trans. Instrum. Meas. 48, 613 (1999).

[8] See, e.g., Atom Interferometry, edited by P. R. Berman (Academic Press, San Diego, 1997).

[9] T. P. Dinneen et al., Phys. Rev. A 59, 1216 (1999).

[10] G. Zinner, T. Binnewies, F. Riehle, and E. Tiemann, Phys. Rev. Lett. 85, 2292 (2000).

[11] Y. Castin, J. I. Cirac, and M. Lewenstein, Phys. Rev. Lett. 80, 5305 (1998).

[12] M. Machholm, P.S. Julienne, and K.-A. Suominen, physics/0103059, 2001.

[13] H. Wallis and W. Ertmer, J. Opt. Soc. Am. B 6, 2211 (1989).

[14] T. Ido, Y. Isoya, and H. Katori, Phys. Rev. A 61, 061403 (2000).

[15] F. Diedrich, J. C. Bergquist, W. M. Itano, and D. J. Wineland, Phys. Rev. Lett. 62, 403 (1989).

[16] W. Rooijakkers, W. Hogervorst, and W. Vassen, Phys. Rev. Lett. 74, 3348 (1995).

[17] Y. Castin, H. Wallis, and J. Dalibard, J. Opt. Soc. Am. B 6, 2046 (1989).

[18] E. A. Curtis, C.W. Oates, and L. Hollberg, physics/ 0104061, 2001.

[19] R. L. Kurucz, Trans. IAU XXB, 168 (1988).

[20] H. Katori, T. Ido, Y. Isoya, and M. Kuwata-Gonokami, Phys. Rev. Lett. 82, 1116 (1999). 